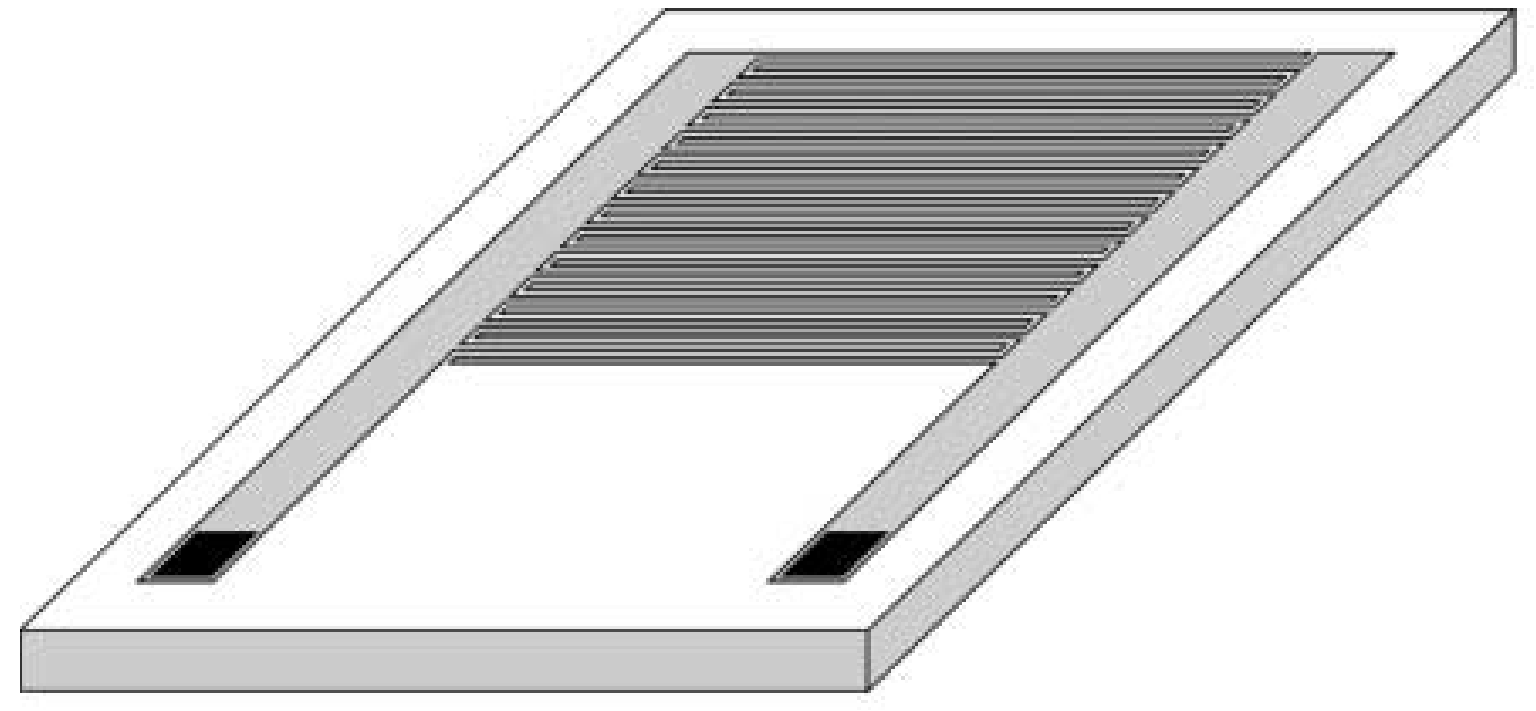

(a)

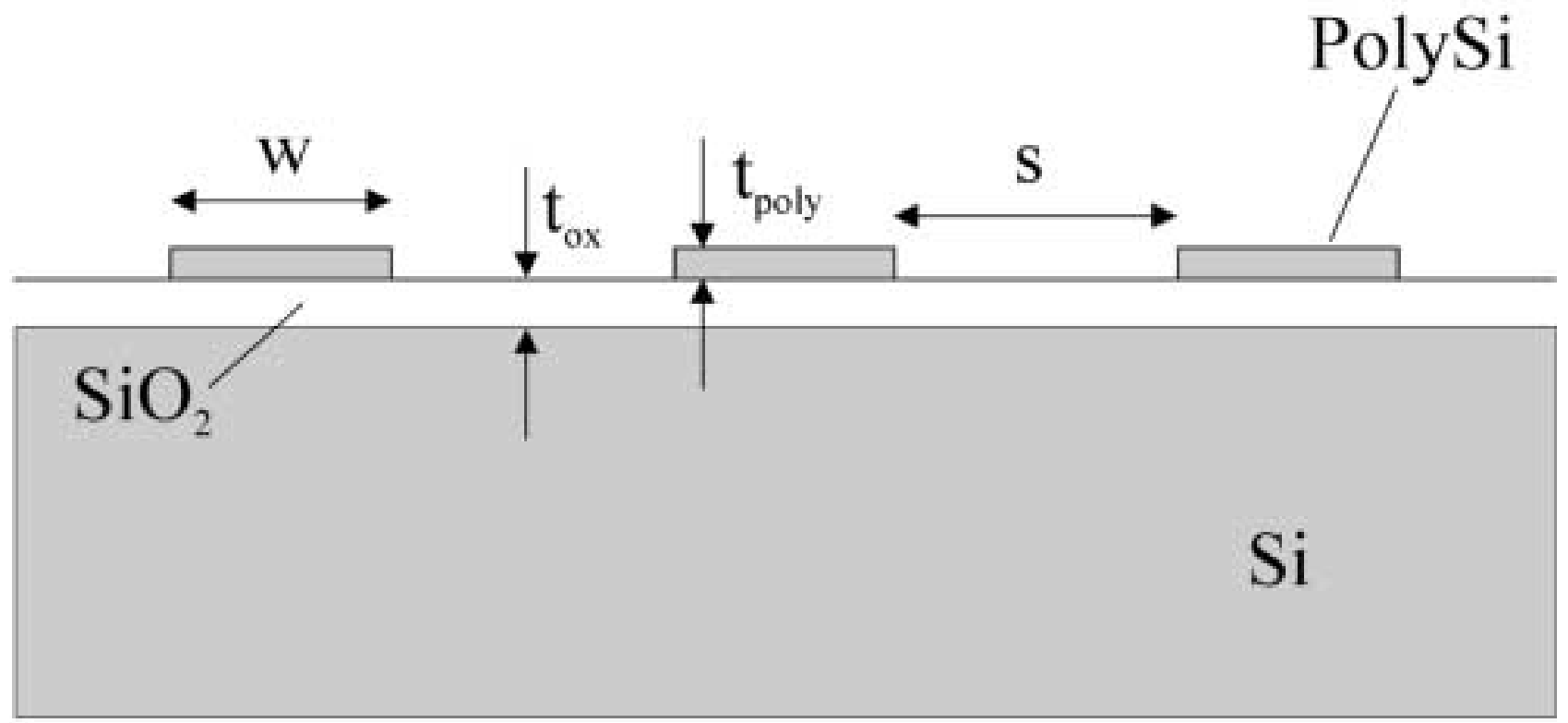

(b) 


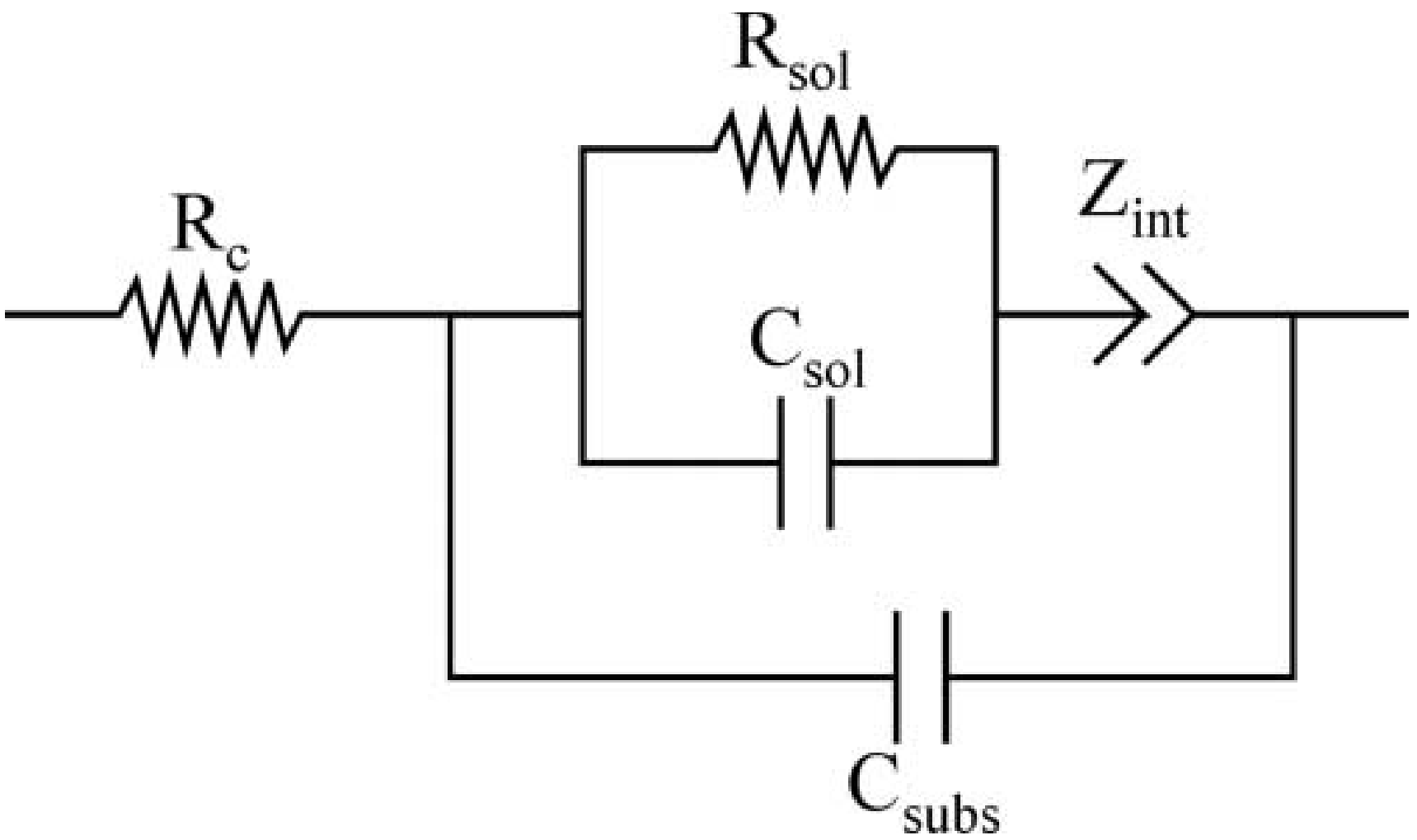


Click here to download high resolution image

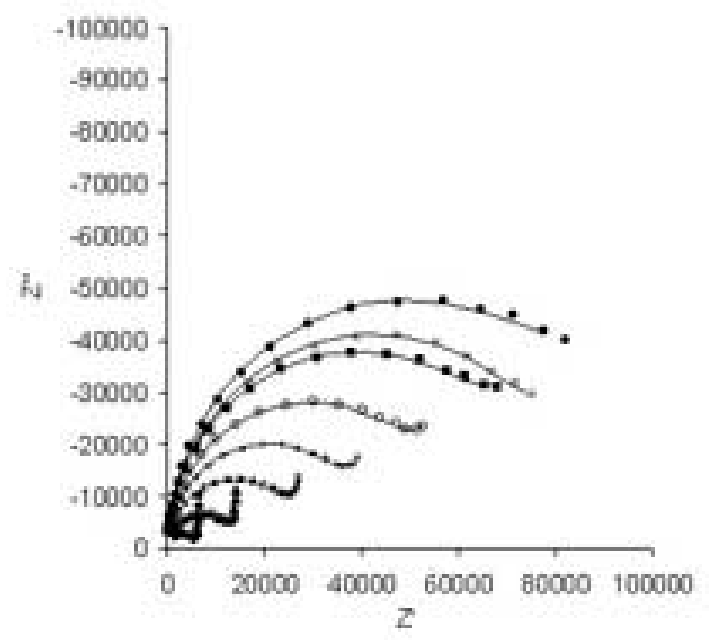


Click here to download high resolution image

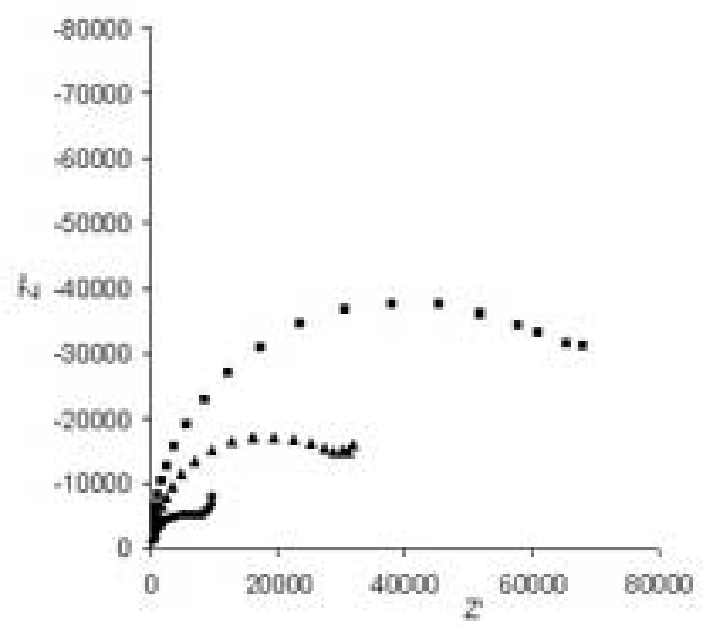




\section{Figure5}

Click here to download high resolution image

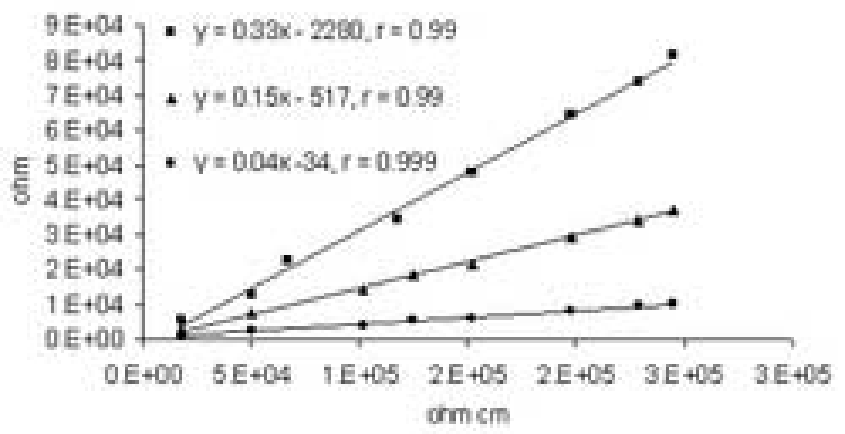




\section{Figure6}

Click here to download high resolution image

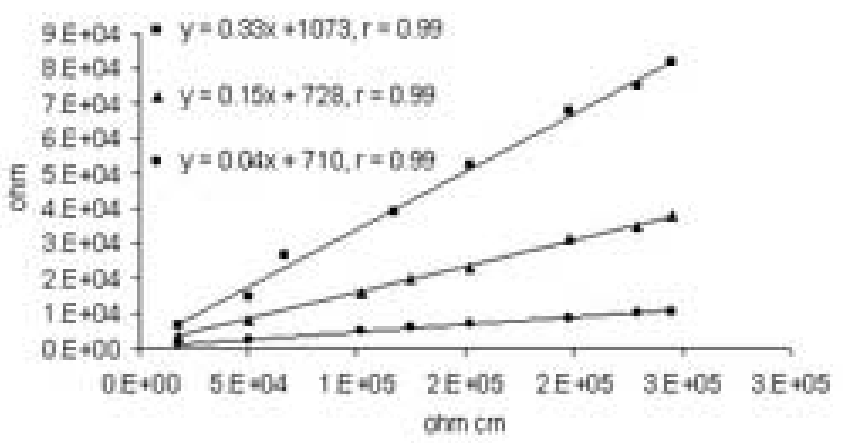



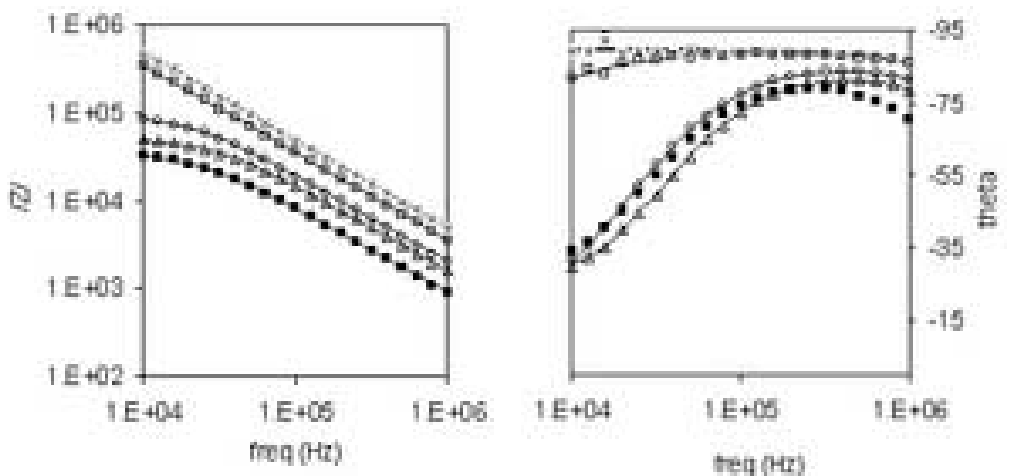
- air
- rexare
s THF
- etranol
a actentrite
- water 
Click here to download high resolution image

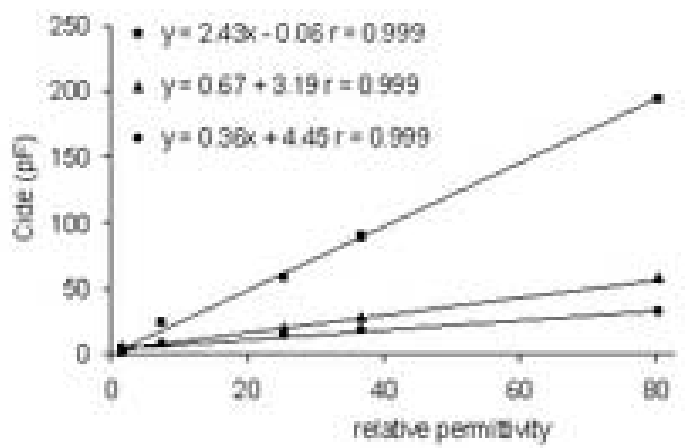




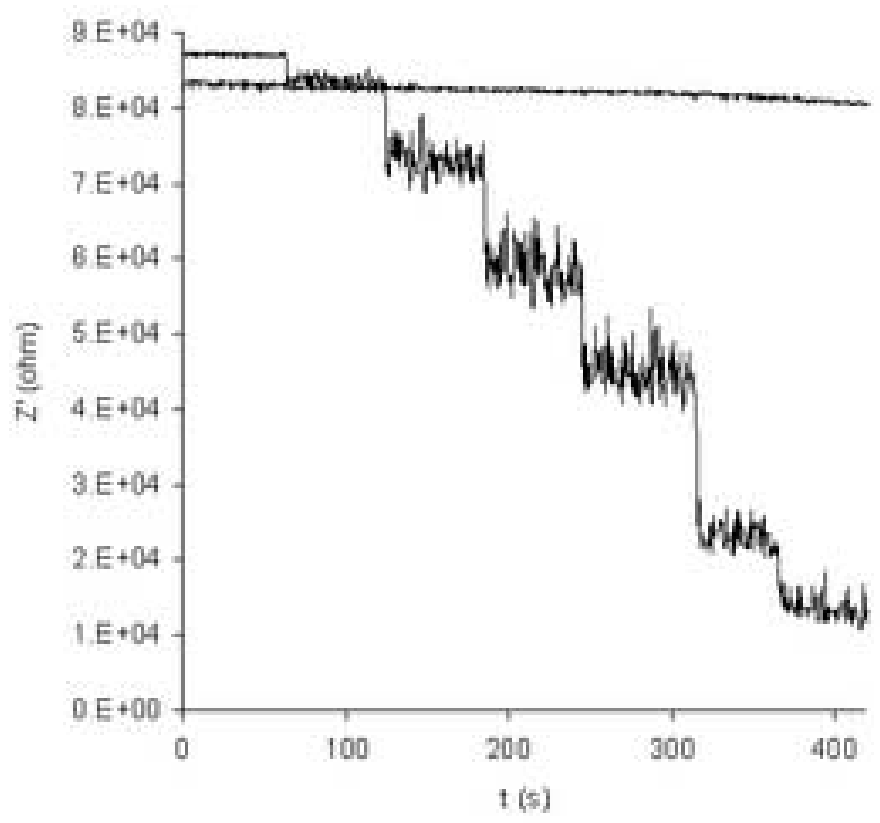


Click here to download high resolution image

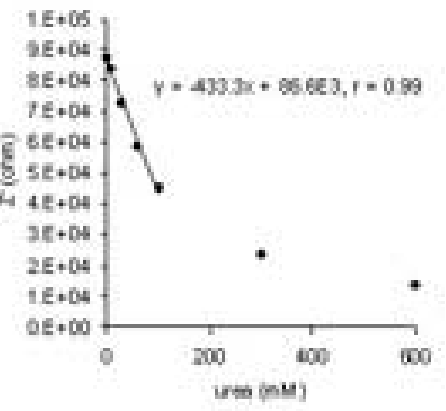




\title{
POLYSILICON INTERDIGITATED ELECTRODES AS IMPEDIMETRIC SENSORS
}

Roberto de la Rica, César Fernández-Sánchez, Antonio Baldi*

Centro Nacional de Microelectrónica (IMB-CNM, CSIC), Campus UAB, 08193 Cerdanyola, Spain, Tel:34 935947700, Fax: 34 935801496, email: Antoni.baldi@.cnm.es

\begin{abstract}
The suitability of polysilicon as a material for the fabrication of interdigitated electrodes and their application to the development of sensors is studied in this work. The main interest in using this material lies in the possibility of obtaining integrated sensors with commercial CMOS technologies and simple post-processing steps. Electrodes with $3 \mu \mathrm{m}$ finger width and 3,10 , and $20 \mu \mathrm{m}$ spacing were fabricated and characterised. Conductivity measurements in the range from 4 to $50 \mu \mathrm{S} / \mathrm{cm}$ yielded a linear response with cell constants of $0.04 \mathrm{~cm}^{-1}, 0.15 \mathrm{~cm}^{-1}$ and $0.33 \mathrm{~cm}^{-1}$, respectively. Permittivity measurements in the range from $\varepsilon_{\mathrm{r}}=80.1$ to $\varepsilon_{\mathrm{r}}=1.89$ yielded a linear response and similar cell constants. The possibility to functionalise both the electrode fingers and the space in between them using a single silanisation process is an interesting advantage of polysilicon electrodes. An urease-based biosensor was obtained with this procedure and characterisation results are reported.
\end{abstract}

KEYWORDS: Interdigitated electrodes, polysilicon, impedimetric sensor, conductometric sensor, biosensor. 


\section{INTRODUCTION}

Planar microelectrodes for imped imetric measurements offer many possibilities for the construction of physical, chemical and "bio-" sensors. Their robust and simple structure, their long term stability and reliability, and easy fabrication have attracted much attention from the sensor research community. Applications that have been proposed for these microelectrodes include measurement of the concentration of calcium [1], potassium [2], $\mathrm{p}(\mathrm{CO} 2)$ [3], $\mathrm{pH}$ [4], ethanol/methanol in gasoline [5], heavy metals [6], nitrate [7], urea [8-9], glucose [10], total prostate-specific antigen (PSA) [11], bacteria [12], red blood cells (hematocrit) [13], and detection of specific sequences of DNA [14]. In some applications, the measurement of a physical magnitude such as dielectric constant or conductivity of the media provides direct information of the analyte concentration $[5,13]$. In other applications, the deposition of a membrane or functional monolayer on top of the electrodes as a recognition element is neces sary to obtain sensitivity and selectivity to particular species [1-4, 6-14].

Different arrangements of electrodes (i.e. different number of electrodes and geometries) may be used for impedimetric measurements. Among them, interdigitated electrodes have significant advantages for certain applications: 1) their low cell constant (resistance to resistivity ratio) permit the measurement of very low conductivity solutions, 2) measurement of dielectric properties is possible thanks to a high inter-electrode capacitance (higher than stray capacitance even for conductive substrates), 3) the short penetration depth 
of electric fields make them less dependent on the measurement cell geometry and allow the use of thin membranes for tailoring selectivity.

In this paper the suitability of polysilicon as a material for the fabrication of interdigitated electrodes and their application to the development of sensors is studied. For example, the feasibility of measuring solution conductivity and permittivity with polysilicon electrodes is demonstrated. The main interest in using this material lies in the possibility of fabricating the electrodes with commercial CMOS technologies and simple post-processing steps, and therefore being able to integrate them as sensors in a more complex system-onchip. Another interesting advantage is that this material can be easily modified following a one-step silanisation reaction. In this way, robust immobilisation of either biomolecules or membranes can be carried out on to both the electrode fingers and the area in between them with the aim of developing a sensor device. As an example, a fully functionalised biosensor for the detection of urea is also presented.

The basic structure of the interdigitated electrodes is depicted in Fig. 1. The polysilicon traces forming the interdigitated geometry are separated from the silicon substrate by a thick silicon dioxide layer. Fig. 2 shows the equivalent circuit of the interdigitated electrodes in aqueous solution. The elements that are repeated for each electrode have been grouped in a single equivalent component. The resistance and capacitance of the solution are represented by $R_{s o l}$ and $C_{\text {sol. }}$. From these components, the resistivity $\rho$ (or the conductivity $\sigma$ ) and the permittivity $\varepsilon$ of the solution can be estimated, respectively. The ratio between measured components and actual physical parameters of the solution, the so-called cell constant, is defined as:

$$
k \equiv R_{s o l} / \rho=\varepsilon / C_{\text {sol }}
$$


The cell constant value is set by the electrode geometrical parameters. There are approximate analytical expressions to calculate the theoretical cell constant corresponding to a particular electrode geometry ( $w$ and $s$ parameters in Fig. 1.b) [15]. However, for the present technology, where the thickness of the polysilicon fingers $\left(t_{p o l y}\right)$ is not negligible compared to their width and spacing, the cell constant is more precisely calculated by Finite Element Analysis (FEA) simulations. In the present work, the low frequency electromagnetic field simulation software Maxwell2D (Ansoft, Inc.) has been used for this aim.

The resistance of the polysilicon traces is represented by $R_{c}$ in the equivalent circuit. Polysilicon is more resistive, even if doped to degeneration, than noble metals typically used for the fabrication of interdigitated electrodes. However, thicker polysilicon layers can be deposited and patterned with standard processes, which partially compensates for the higher resistivity. The capacitance of the electrodes to the substrate is represented by $C_{s u b s}$, and its value depends on the thickness of the silicon dioxide layer isolating the electrodes from the silicon substrate. $R_{c}$ and $C_{\text {subs }}$ limit the minimum resistivity and permittivity that can be measured and should be minimised in the design.

The impedance of the interface between the polysilic on and the solution is represented in the equivalent circuit by $Z_{\text {int }}$. For a typical metal electrode in the presence of an electroactive compound, the interface impedance is represented by a Randless circuit. This circuit is composed of the double layer capacitance in parallel with the impedance of the charge transfer processes, the so-called faradic impedance. In the case of polysilicon electrodes the impedance of the native oxide layer has to be added in series. This layer spontaneously grows on top of the polysilicon structures in contact with air. The presence of the native silicon oxide passivates the electrode surface, thereby obstructing the charge transfer processes between the polysilicon and electroactive compounds present in the interface. As a consequence, these electrodes seem to be unsuitable for electrochemical 
impedance measurements and the possibility to use them for that purpose has not been further studied. In the equivalent circuit presented here the whole interface impedance has been associated to a constant phase element:

$$
Z_{\text {int }}=\frac{1}{\left(j \omega C_{C P E}\right)^{\alpha_{C P E}}}
$$

With the aim of studying the appropriateness of the proposed equivalent circuit, three different interdigitated electrode designs have been tested in this work. Every design has the same sensor area but different spacing between fingers, and therefore different number of fingers. The finger width and length is $3 \mu \mathrm{m}$ and $1600 \mu \mathrm{m}$, respectively. The spacing between fingers is 3,10 and $20 \mu \mathrm{m}$, with a total of 218,101 and 57 fingers for each layout. The solution resistance, $R_{\text {sol }}$, should increase with decreasing number of fingers, and therefore the cell constant should also increase.

\section{EXPERIMENTAL}

\subsection{Electrode fabrication}

Fabrication starts with the thermal oxidation of a silicon wafer to form a $1.5 \mu \mathrm{m}$-thick silicon oxide layer. Next, a $0.48 \mu \mathrm{m}$-thick polysilicon layer is deposited by LPCVD, and doped with phosphor to degeneration by diffusion. The polysilicon layer is then patterned with standard photolithography and reactive ion etching. Subsequently, interconnect pads are formed on top of the resulting polysilicon electrodes by deposition and patterning of a $0.5 \mu \mathrm{m}$ thick aluminium layer. Finally, a passivation coating consisting of $3000 \AA$ of silicon oxide and $7000 \AA$ of silicon nitride is deposited and patterned. In this latter step the passivation is removed from the pads and from the interdigitated area. A BOE (Buffered Oxide Etch) 
solution is used to etch the silicon oxide layer because of its high selectivity towards this material, which ensures that polysilicon fingers are left intact.

\subsection{Materials}

Urease from Jack Beans, urea, glycine, (3-aminopropyl)trimethoxysilane and glutaraldehyde were purchased from Sigma-Aldrich (Spain). All other chemicals were of analytical grade. Deionised water (resistivity $>18 \mathrm{M} \Omega$ ) was used throughout this work.

\subsection{Impedance measurements}

All the impedance spectra were taken in the range between $1 \mathrm{MHz}$ and $10 \mathrm{kHz}$ with a SI 1260 SOLARTRON Impedance Analyzer, at 0V DC potential and 10mV AC peak-to-peak amplitude. The experiments were performed with electrodes of each geometry $(3 \times 3,3 \times 10$, $3 \times 20$ nomenclature will be used to refer to electrodes with $w=3$ and $\mathrm{s}=3,10$, and 20 microns, respectively). Impedance spectra were fitted with the aid of the Zview2 software to the equivalent circuit shown in Fig. 2. The goodness of the fittings can be evaluated from the square of the standard deviation between the original data and the calculated spectrum, the socalled $\chi^{2}$ value. All the fittings had a $\chi^{2}$ lower than $10^{4}$.

\subsection{Urea biosensor fabrication.}

$3 \times 20$ electrodes were cleaned by immersing them in a stirred $2.5 \% \mathrm{KOH}$ solution for 30min. APTMS was gas-phase deposited onto the chip surface by keeping the sensor close to the silane in a sealed chamber previously purged with nitrogen. This protocol avoids the formation of a thick layer of APTMS onto the silicon oxide [16]. The electrodes were left to react with the silane overnight, then rinsed with water and dried under a nitrogen stream. 
Subsequently, urease was covalently attached to the sensor surface using glutaraldehyde as a linker between the silanised surface and the enzyme. Thus, the modified electrode was kept in a stirred $8 \%$ glutaraldehyde solution in PBS buffer $\mathrm{pH} 7$ for one hour. Then, it was rinsed with deionized water, dried, and dipped in a $10 \mathrm{mg} / \mathrm{ml}$ urease solution in PBS overnight.

\section{RESULTS AND DISCUSSION}

\subsection{Conductivity measurements}

Solutions with different conductivities in the range from $4 \mu \mathrm{S} / \mathrm{cm}$ to $50 \mu \mathrm{S} / \mathrm{cm}$ were prepared by diluting a concentrated $\mathrm{KCl}$ solution and measuring the final conductivity with a CRISON $\mu$ CM 2202 Conductimeter. The impedance spectra of a $3 \times 20$ sensor immersed in these solutions are shown in Fig. 3. Solutions with a lower conductivity have a larger semicircle (higher $R_{s o l}$ ). It can be seen that a good agreement between real and fitted spectra was achieved, thus validating the proposed equivalent circuit.

A comparison of the three assayed geometries for an $11.3 \mu \mathrm{S} / \mathrm{cm} \mathrm{KCl}$ solution can be seen in Fig. 4. $3 \times 20$ electrodes show higher $R_{\text {sol }}$ than $3 \times 10$ electrodes and $3 \times 10$ electrodes higher $R_{s o l}$ than $3 \times 3$ electrodes. This indicates that the $3 \times 20$ electrodes have a smaller cell constant than $3 \times 10$ and $3 \times 3$ ones, as was expected from the fact that each geometry has a lesser amount of fingers. Table 1 summarises the values for the different equivalent circuit elements and different geometries at this particular conductivity. The theoretical values obtained from FEA simulations for $R_{s o l}$ and $C_{\text {sol }}$ are also included and show a reasonably good agreement with the experimental ones. The difference of about $10 \mathrm{pF}$ between measurement and simulation values of $C_{s o l}$ is probably associated to a parasitic capacitance not taken in account in the simulations. 
$R_{\text {sol }}$ values for the different conductivities assayed were also obtained from the fitting and plotted versus the inverse of the conductivity (resistivity). A linear relationship was obtained. The slope of these curves is the electrodes cell constant. Results for each geometry are shown in Fig. 5. The cell constant of the $3 \times 20,3 \times 10$ and $3 \times 3$ electrodes is $0.33 \mathrm{~cm}^{-1}$, $0.15 \mathrm{~cm}^{-1}$ and $0.04 \mathrm{~cm}^{-1}$, respectively. Alternatively, the real part of the measured impedance at a fixed frequency (Z') was plotted versus resistivity. This frequency was chosen to be at the local minimum of the Nyquist plot (between the semicircle and the low frequency tail), were the real part of the impedance approximates to $R_{\text {sol }}$. Results are shown in Fig. 6, with Z' measured at $10 \mathrm{kHz}$ for the $3 \times 20$ electrodes, $12.6 \mathrm{kHz}$ for $3 \times 10$ and $20 \mathrm{kHz}$ for $3 \times 3$. A linear relationship between Z' and the resistivity can be observed. It is also important to note that the cell constant obtained with this methodology is the same as the one obtained from fitting the whole curve.

\subsection{Permittivity measurements}

In order to prove that the polysilicon interdigitated electrodes are suitable for the detection of changes in the permittivity of the medium, impedance spectra were taken in different solvents as well as in air. The assayed solvents were water $\left(\varepsilon_{r}=80.1\right)$, acetonitrile ( $\left.\varepsilon_{r}=36.6\right)$, ethanol $\left(\varepsilon_{r}=25.3\right)$, tetrahydrofuran $\left(\varepsilon_{r}=7.52\right)$ and hexane $\left(\varepsilon_{r}=1.89\right)$. Fitted plots were obtained as above. Bode plots for the real and fitted spectra of $3 \times 3$ electrodes are shown in Fig. 7. $C_{\text {sol }}$ was plotted versus $\varepsilon$ after subtracting $C_{\text {subs }}$, which was obtained by measuring the electrodes in air. Results are shown in Fig. 8 where a linear relationship between $C_{\text {sol }}$ and $\varepsilon_{r}$ was found for all geometries. The cell constant values obtained from capacitive measurements were $0.04,0.13$, and $0.25 \mathrm{~cm}^{-1}$ for the $3 \times 3,3 \times 10$ and $3 \times 20$ electrode geometries, 
respectively. Good agreement was observed with cell constant values obtained from resistive measurements.

\subsection{Urea enzymatic sensor}

The capability of the fabricated biosensor to detect changes in the concentration of urea was assayed. The sensor was immersed in a stirred $50 \mathrm{mM}$ glycine solution pH 5.7 (conductivity $3.7 \mu \mathrm{S} / \mathrm{cm}$ ) and the impedance measured at a set frequency of $10 \mathrm{kHz}$. The reaction catalysed by urease is described elsewhere [17]. The sensor response to the urea concentration was tested by adding the right amount of a $0.1 \mathrm{M}$ urea stock solution every 60 seconds. Plain $3 \times 20$ electrodes were used as a blank. Results of the changes in Z' with time are depicted in Fig. 9. The relationship between Z' and the urea concentration was found to be linear in the range between $10 \mu \mathrm{M}$ and $100 \mu \mathrm{M}$, with a sensitivity of $-433.3 \Omega / \mu \mathrm{M}$ (Fig.10).

\section{CONCLUSIONS}

Polysilicon interdigitated electrodes with different geometries were fabricated and characterised. Linear responses were obtained when used to measure conductivity and permittivity. The cell constants obtained from characterisation showed good agreement with the theoretical ones obtained from simulations. An urea biosensor based on the immobilisation of urease on the electrodes surface was also characterised. A sensitivity of $433.3 \Omega / \mu \mathrm{M}$ was obtained. This results show that polysilicon is a suitable material for the fabrication of interdigitated electrode-based impedimetric sensors.

Polysilicon interdigitated electrodes with different geometries were fabricated and characterised. In particular, the suitability of these electrodes to measure both the conductivity and permittivity of low ionic strength solutions was demonstrated. Linear 
responses were obtained in both cases and the cell constants showed good agreement with the theoretical ones obtained from simulations. In the case of conductivity measurements, it was also proved that measuring the real part of the impedance at an appropriate frequency also yielded a linear response, no further treatment of data being necessary to obtain this parameter. This feature is of particular interest when developing sensors devoted to real-time measurements, i.e. in flow systems.

Finally, an application of the proposed electrode arrangement to the fabrication of a conductometric urea biosensor was developed. It was proved that an urease-based sensor can be developed by covalently attaching the enzyme to the previously silanised sensor surface. The measurement of the impedance at a fixed frequency enabled the real-time monitoring of urean concentratin.

\section{ACKNOWLEDGMENTS}

Funding for this project was provided by the Ministry of Education and Science of the Spanish government (ref. TEC2004-00068/MIC).

\section{REFERENCES}

[1] U. Trebbe, M. Niggemann, K. Cammann, G. C. Fiaccabrino, M. Koudekka-Hep, S. Dzyadevich, O. Shulga, Fresenius J. Anal. Chem. 371 (2001) 734

[2] A.E. Shvarev, D.A. Rantsan, K.N. Mikhelson, Sens. Act. B 76 (2001) 500

[3] A. R. Varlan, W. Sansen, Sens. Act. B 44 (1997) 309

[4] N.F. Sheppard Jr., M.J. Lesho, P. McNally, A.S. Francomacaro, Sens. Act. B 28 (1995) 95

[5] T. Hofmann, F. Bcckmann, S. Michaelis, J. Zacheja, J. Binder, S. Tagliante, Sens. Act. A $61(1997) 319$ 
[6] C. Chouteau, S. Dzyadevych, C. Durrieu, J.M. Chovelon, Biosens. Bioelectron. 21 (2005) 273

[7] X. Wang, S.V. Dzyadevych, J.M. Chovelon, N. Jaffrezic-Renault, L. Chen, S. Xia, J. Zhao, Electrochem. Commun. 8, (2006) 201

[8] P. Jacobs, J. Suls, W. Sansen, Sens. Act. B 20 (1994) 193

[9] A. Steinschaden, D. Adamovic, G. Jobst, R. Glatz, G. Urban, Sens. Act. B 44 (1997) 365

[10] S.V. Dzyadevicha, V.N. Arkhipova, A.P. Soldatkina, A.V. El'skaya, A.A. Shul'ga, Anal. Chim. Acta 374 (1998) 11

[11] C. Fernandez-Sanchez, C. J. McNeil, K. Rawson, O. Nilsson, Anal. Chem 76 (2004) 5649

[12] N. Kordas, Y. Manoli, W. Mokwa, M. Rospert, Sens. Act. B 43 (1994) 31

[13] A.R. Varlan, P. Jacobs, W. Sansen, Sens. Act. B 34 (1996) 258

[14] L. Moreno-Hagelsieb, P.E. Lobert, R. Pampin, D. Bourgeois, J. Remacle, D. Flandre, Sens. Act. B 98 (2004) 269

[15] W. Olthuis, W. Streekstra, P. Bergveld Sens. Act. B 24-25 (1995) 252

[16] Janildo L. Magalhaes, Leonardo M. Moreira, Ubirajara P. Rodrigues-Filho, Martha J. Giz, Marcelo A. Pereira-da-Silva, Richard Landers, Rita C. G. Vinhas, Pedro A. P. Nascente. Surf. Interface Anal. 33 (2002) 293

[17] Lee,W.Y., Lee,K.S., Kim,T.H., Shin,M.C., Park,J.K. Electroanalysis 12 (2000) 78 


\section{TABLES}

Table 1

Summary of equivalent circuit element values obtained from fitting of the experimental curves and from FEA simulations for $11.3 \mu \mathrm{S} / \mathrm{cm}$

\begin{tabular}{ccccccccc}
\hline Geometry & $\begin{array}{c}R_{\text {sol }}(\Omega) \\
\text { measured }\end{array}$ & $\begin{array}{c}R_{\text {sol }}(\Omega) \\
\text { simulated }\end{array}$ & $\begin{array}{c}C_{\text {sol }}(\mathrm{pF}) \\
\text { measured }\end{array}$ & $\begin{array}{c}C_{\text {sol }}(\mathrm{pF}) \\
\text { simulated }\end{array}$ & $R_{c}(\Omega)$ & $\begin{array}{c}C_{\text {subs }} \\
(\mathrm{pF})\end{array}$ & $\begin{array}{c}\mathrm{C}_{\mathrm{CPE}} \\
(\mathrm{nF})\end{array}$ & $\alpha_{\mathrm{CPE}}$ \\
\hline $3 \times 3$ & 3506 & 3918 & 180 & 163 & 267 & 32 & 9.5 & 0.88 \\
$3 \times 10$ & 12757 & 14598 & 54 & 44.3 & 256 & 26 & 5.8 & 0.88 \\
$3 \times 20$ & 26924 & 33496 & 30 & 19.7 & 197 & 23 & 23 & 0.73 \\
\hline
\end{tabular}




\section{FIGURE CAPTIONS}

Figure 1. Schematic representation of the interdigitated electrodes layout (a) and their crosssection (b). Geometrical parameters are indicated.

Figure 2. Equivalent circuit of the interdigitated electrodes immersed in a solution.

Figure 3. Nyquist plot of $3 \times 20$ electrodes immersed in $\mathrm{KCl}$ solutions of different conductivity. Dotted plots are the empiric impedance spectra; solid lines correspond to the fittings.

Figure 4. Nyquist plot for $3 \times 3$ (circles), $3 \times 10$ (triangles) and $3 \times 20$ (squares) electrodes immersed in a $\mathrm{KCl}$ solution of conductivity $11.3 \mu \mathrm{S} / \mathrm{cm}$.

Figure 5. Resistivity $v s$. solution resistance plot for the three geometries assayed $(3 \times 3$ circles, $3 \times 10$ triangles, $3 \times 20$ squares $)$.

Figure 6. Resistivity $v s . Z^{\prime}$ at a fixed frequency for the three geometries assayed $(3 \times 3$ circles, $3 \times 10$ triangles, $3 \times 20$ squares). The frequencies were $10 \mathrm{kHz}$ for $3 \times 20,12.8 \mathrm{kHz}$ for $3 \times 10$ and $2.5 \mathrm{kHz}$ for $3 \times 3$.

Fig 7. Bode plots of $3 \times 3$ electrodes immersed in solvents with different permittivity. Dotted plots are the empiric impedance spectra; solid lines correspond to the fittings. 
Figure 8 . Permittivity vs. $C_{s o l}$ for the three geometries assayed ( $3 \times 3$ circles, $3 \times 10$ triangles, $3 \times 20$ squares).

Figure 9. Changes in Z'vs. time upon the addition of urea for plain electrodes and ureasemodified electrodes ( $3 \times 20$ electrodes).

Figure 10. Urea concentration $v s$. Z' plot for the proposed biosensor. 